\title{
Design and implementation of multimedia player on embedded platform
}

\author{
Zhang xijun; Xue jianbin; Lin ying; He jiai \\ Department of Computer and Communication Lanzhou University of Technology, Gansu Province, China \\ zxjun@lut.cn
}

\begin{abstract}
The terminal based on the embedded technology of S3C6410 and ARM Linux is widely used in many fields. We study the system of embedded system. The product is mainly included video playing, music playing and picture display as like MPlayer. The software run on the platform of Fedoral2 and QT/Embedded will be the main topic of this paper. This paper is focused on the embedded platform, then discuss the hardware of the system including the design plan of hardware, core board level interface, expanded plate circuit and systemic function. After that this paper will make a detailed analysis of the environment of system and design of system functions, which include ARM Linux system structure, pictures, video player and music player. At last this paper will give the test result of all system functions.

Index Terms- ARM Linux; S3C6410; Multimedia terminal; QT/Embedded
\end{abstract}

\section{Introduction}

With the rapid development of the embedded chip technology the function of the realization of the embedded system is more and more abundant and the system performance is becoming more and more perfect. Nowadays the embedded system has gradually been into the people's daily life. It is bringing a great convenience, and has a profound effect on the world. In addition to playing multimedia file embedded multimedia system also supports many functions such as the picture browsing, electronic books, taking pictures, videos, games and the radio.

In the view of the foreign the PMP design is more mature than the current domestic PMP design programs, so this paper is mainly discussed a way of low-cost embedded processor. It is used the higher cost ARM11 chip multimedia terminal design. It can display the common picture files and play a variety of popular audio and video files. There are three mature solutions of the internal structure of the embedded multimedia terminal, but they have their own shortcomings [1]. Through the analysis of the functional requirements of the system we choose the ARM11 processor based on the S3C6410 UPNETARM2410-S embedded development platform as a hardware development platform and the ARMLinux operating system and QT as a software development platform.

\section{The system hardware platform design and implementation}

The system block diagram is given as the Fig.1. It is made of three layers. The lower layer is the system layer. It includes the driver of the interface unit and it can provide service to the upper layer. The middle layer is the interface layer. It is the interface layer of the embedded GUI. The upper layer is the oriented application layer, it provides software interface to the people. User can use many services through the touch screen. In this layer it includes picture browsing module, audio playback module and video broadcast module and etc.

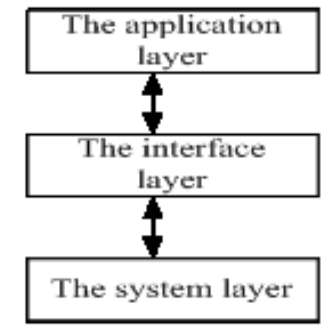

Fig.1 System structure diagram

\section{ARM Linux system construction}

Embedded multimedia terminal is designed based on ARM Linux operating system. ARM Linux is a small operating system. It is one of the supported operating system software for embedded system applications. It is the most important part in embedded systems (including software systems and hardware systems). It is usually contained the underlying hardware driver system kernel, communication protocols, device interface driver, standardization of browser and graphical interface and etc. It is shown in the Fig.2.

\begin{tabular}{|c|c|c|c|c|}
\hline \multicolumn{5}{|c|}{ Application program } \\
\hline $\begin{array}{c}\text { Network } \\
\text { Interface } \\
\text { module }\end{array}$ & $\begin{array}{c}\text { Memory } \\
\text { Driver } \\
\text { module }\end{array}$ & $\begin{array}{c}\text { GPIO } \\
\text { Driver } \\
\text { module }\end{array}$ & $\begin{array}{c}\text { Audio } \\
\text { Output } \\
\text { Driver } \\
\text { module }\end{array}$ & $\begin{array}{l}\text { Graphic } \\
\text { Driver } \\
\text { module }\end{array}$ \\
\hline \multicolumn{5}{|c|}{ ARM Linux system kernel } \\
\hline \multicolumn{5}{|c|}{ S3C6410 hardware platform } \\
\hline
\end{tabular}

Fig.2 ARM Linux system structure

\section{ARM Linux system structure}

Usually there are two ways to build cross-compiler environment for ARM Linux developers. One is to use compiled development environment which can be unlocked 
the installation in the corresponding position; the other is that the people download the appropriate source code of the program and then compile and create a development environment. In this paper I use the first method to create a development environment. Firstly I install Fedora 12 operating system on the PC and then download the precompiled crosscompilation environment arm920t-eabi.tgz and cross2.95.3.tar.bz2. Arm-linux-gcc-4.3.1, 4.3.2 is used to compile the kernel, 2.95 .3 is used to compile vivi. The 2.95.3's installation position is put in /user/local/arm down. Then we can use the arm-Linux-GCC - $v$ to view whether the cross compiler current version is 2.95.3. If it is, the result is proved successfully, otherwise it is failure.

\section{The realization of the Embedded graphic interface}

The embedded multimedia terminal software platform uses the QT/Embedded. QT is one of the most development tools which are used to develop GUI application in embedded development. The development of embedded applications program is completed on a PC. The output results are displayed in a simulation of a small device display terminal emulator, when we develop embedded applications on a PC. The application program is compiled and linked into binary object code which is suitable for running on the hardware platform to achieve stable operation on the embedded hardware platform. In addition, due to the application of this work is developed in QT/Embedded platform, it also need the support of the QT/Embedded library. Embedded application program diagram based on the QT/Embedded is shown in Fig.3.

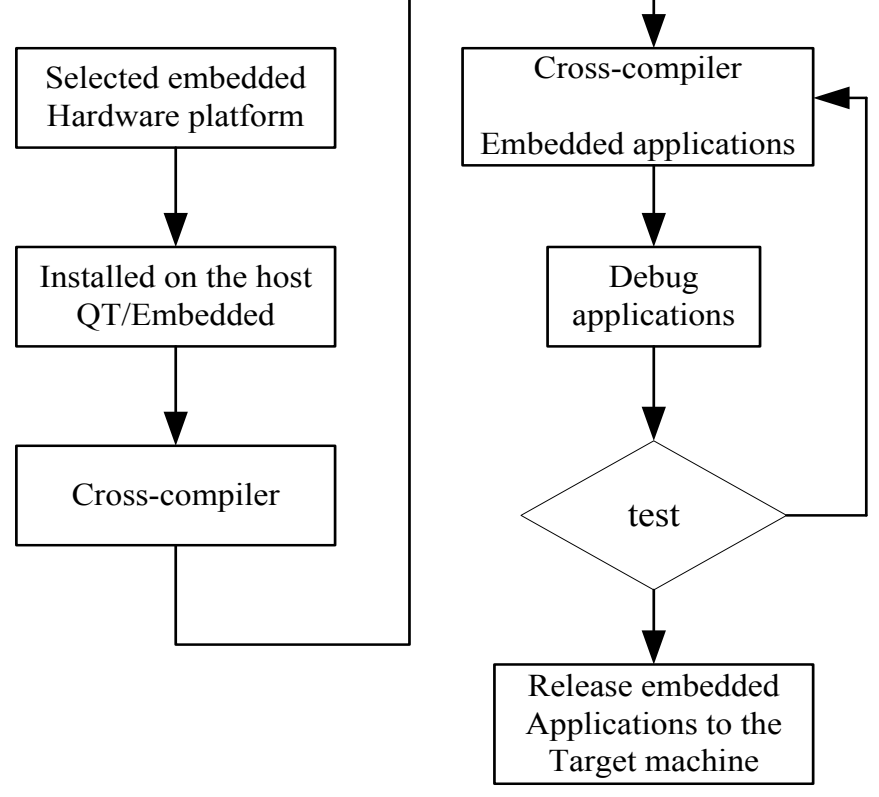

Fig.3 QT/Embedded application development flow chart

\section{A. The realization of the embedded audio player}

According to the above introduction, the basic function of the PC audio player based on Fedora12 operating system is implemented, but it is only for PC. Thus it needs to be transplanted into s3c6410 development platform. It needs to use ARM/QT to recompile and modify the audio player of parameters. The way of compiling ARM audio player method is the same with the pictures player. The process is shown in Fig.4.

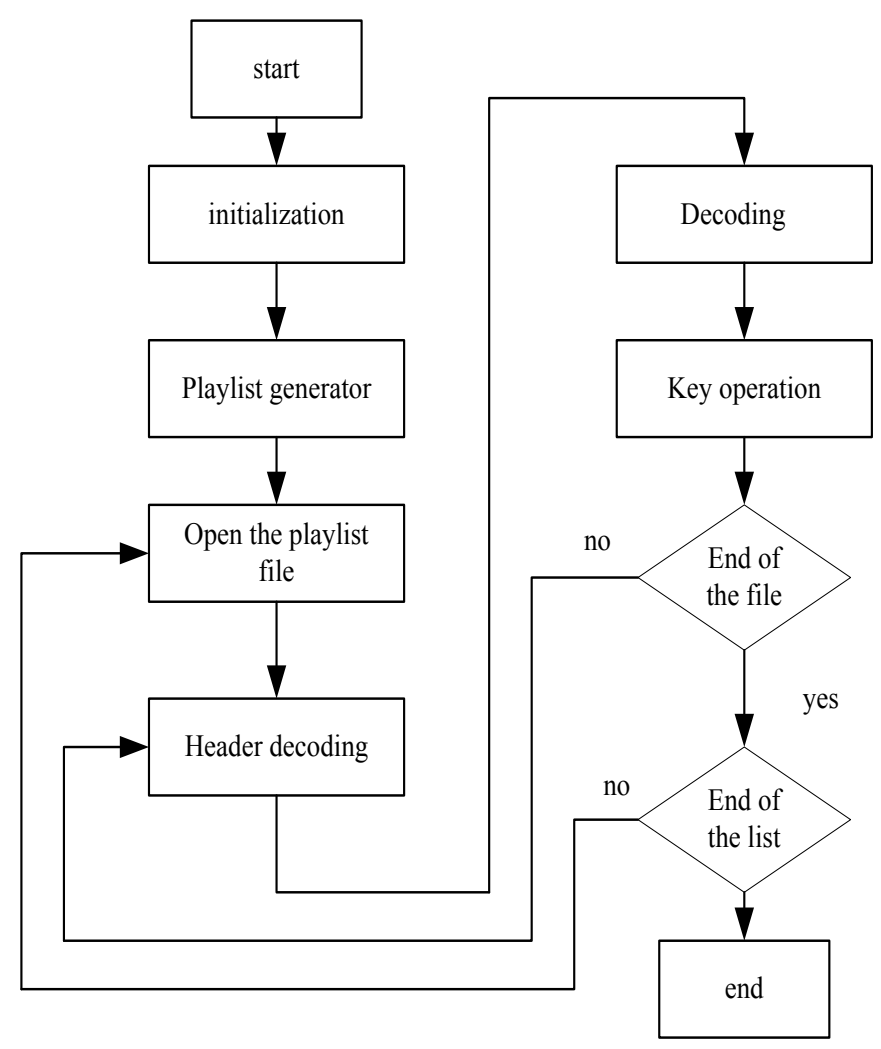

Fig.4 audio player integral control flow chart

\section{B. Test of the Video file playback function}

We use the Qt4.6.3-arm to compile the video file player project and at the same time set parameters of -qws. After the completion of the compiler will generate, the binary file is downloaded to the development platform for testing [2-4]. Run the binaries of the video file at video player, s3c6410 development platform will display video file player interface, which is shown in Fig.5. Removable storage device is plugged into the s3c6410 development platform. Remove storage devices are stored in various formats of video files, such as: rmvb, avi, rm, etc. For video player specific test is as follows:

(1) video format supported by the video player test. Read the mobile storage equipment of video files, check whether to support the RMVB, avi, rm,etc.Kinds of the current mainstream video file format.

(2) each video player function button test: Read the video files in the mobile storage equipment. Realize playlist display through the play list on video file specified broadcast. Testing the volume adjustment, pause, recovery, restart, add upcoming video files and delete video file function while playing the video file.

(3) interactive test: use the touch screen or USB, keyboard, mouse to complete the video player test. 


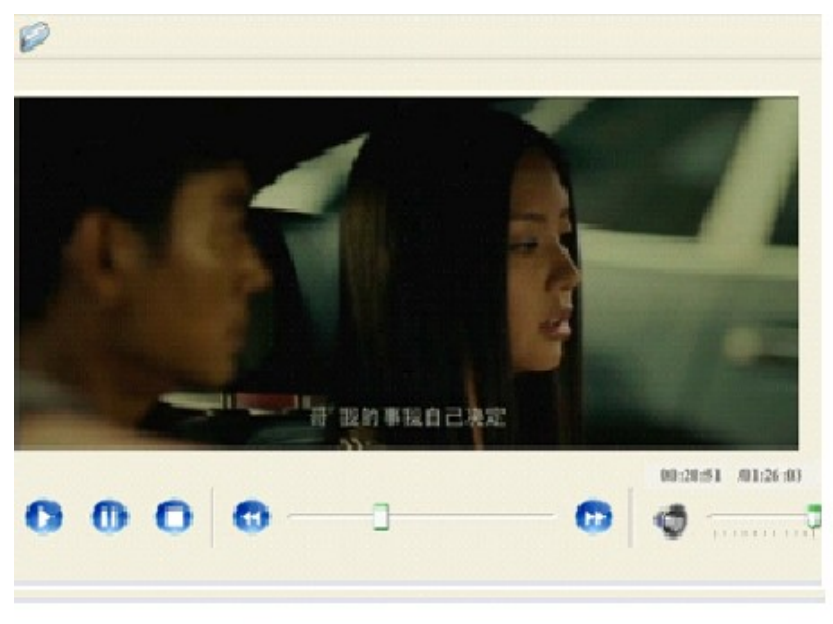

Fig.5 embedded video player

\section{Conclusion}

Test process is as follows:

(1)Target machine and the host machine is linked together, build cross-compile environment. We can download the pictures and video player, audio player executable binary file to the target machine.
(2) We start the target machine and control the program of the target machine through the super terminal. Using the completed shell scripting to start embedded multimedia terminal program and insert $U$ disk to the target machine.

(3) Operating picture file player and testing each function module. Closing the picture file player and running audio file player and test various functional modules.

(4)Observe the super terminal output results in multimedia terminal read multimedia files.

\section{Acknowledgment}

Project supported by the National Natural Science Foundation (No. 61062002), Lanzhou university of Tamarisk youth teacher Foundation(No.Q201215) and Gansu Natural Science Foundation(2011GS04175).

\section{References}

[1] M. Varchola, M. Drutarovsky. Embedded Platform for Automatic Testing and Optimizing of FPGA Based Cryptographic True Random Number Generators. Radio engineering. 2009. Vol.18. No.4. Pp:631-638.

[2] Fugang Duan;Zhan Shi. The Implement of MPEG-4 Video Encoding Based on NiosII Embedded Platform. Journal of Capital Institute of Physical Education. 2009, Vol.3. No.3.

[3] A. Gomez-Perez, M. Fernandez-Lopez and O. Corcho, Ontological Engineering: With Examples from the Areas of Knowledge Management, e-Commerce and the Semantic Web, Springer-Verlag, New York, 2003.

[4] K. Lane et al., "Validation of synthetic aperture radar for iceberg detection in sea ice," in Proc. IGARSS '04, Vol.1, pp 125-128, 2004. 